Research Article

\title{
The Diagnostic Method for Open-Circuit Faults in Inverters Based on Extended State Observer
}

\author{
Chaobo Chen, ${ }^{1}$ Ying Yang, ${ }^{2}$ Binbin Zhang, ${ }^{2}$ and Song Gao $\mathbb{D}^{2}$ \\ ${ }^{1}$ School of Automation and Information Engineering, Xi'an University of Technology, Xi'an 710048, Shaanxi Province, China \\ ${ }^{2}$ School of Electronic Information Engineering, Xi'an Technological University, Xi'an 710021, Shaanxi Province, China \\ Correspondence should be addressed to Song Gao; gaos@xatu.edu.cn
}

Received 21 February 2021; Revised 9 April 2021; Accepted 15 April 2021; Published 26 April 2021

Academic Editor: Ahmed G. Radwan

Copyright $(0) 2021$ Chaobo Chen et al. This is an open access article distributed under the Creative Commons Attribution License, which permits unrestricted use, distribution, and reproduction in any medium, provided the original work is properly cited.

To reduce the influence of unknown disturbance on open-circuit fault diagnosis of inverters in the motor drive system, an opencircuit fault diagnosis method, which is based on extended state observer, is proposed for inverters. A mixed logic dynamic model of the inverters is established by analyzing the current flow path when the system works normally and there are open-circuit faults. A voltage extended state observer is designed for the mixed logic dynamic model. The open-circuit faults are detected according to the phase voltage residual between the observed voltage and the actual voltage. The position of the faulty switches is determined by querying the voltage residual information table. Finally, the simulation results show that the method can effectively reduce the influence of the unknown interference on the inverter faults diagnosis, improve the fault diagnosis rate, and verify the effectiveness and feasibility of the method.

\section{Introduction}

With the rapid development of power electronics technology, motors are widely used in industry, military, aviation, and so on, and many motors are driven by inverters. In reality, if the inverter fault cannot be detected in time, it will directly affect the operation of the motor drive system and even the safety of the whole system. Therefore, fast fault diagnosis of the inverter is of great significance to improve the reliability of the system.

Inverter faults are mainly caused by power electronic fault, and $38 \%$ of the inverter faults are IGBT (Insulated Gate Bipolar Transistor) fault [1]; there is short-circuit or opencircuit fault about IGBT fault. Short-circuit fault occurs for a short period and cannot be diagnosed by software algorithms. Inverter short-circuit fault can transform into an open-circuit fault due to the break of the connecting fast fuses in the circuit, so the short-circuit fault can be diagnosed by the open-circuit faults diagnostic method [2]. At present, the diagnostic methods of IGBT open-circuit fault are classified into the current-based method, the voltagebased method, and the intelligent algorithm methods. The intelligent inverter fault diagnosis methods are mainly the neural network method [3-5], the fuzzy logic method [6], spectrum analysis method [7], and so on. Due to these intelligent fault diagnosis methods which rely on experience and the fact that they are subjective, they are less used in inverter fault diagnosis. The current-based inverter fault diagnosis methods are mainly the current vector trajectory slope method [8], the current vector instantaneous frequency method [9], average current Park's vector approach [10], and so forth. However, the current vector trajectory slope method is susceptible to load changes, the current vector instantaneous frequency method only detects the open-circuit fault, and average current Park's vector approach will be misdiagnosed if the threshold is unsuitable. The inverter fault voltage-based methods are mainly the voltage analytical model method [11], switching function model-based method [12], mixed logic dynamic model method [13-15], and so forth. In [12], the switching function model-based method is studied, but it is easy to cause misdiagnosis because the dead time of the switch devices is not considered. Moreover, this method requires a highspeed optocoupler or comparator when diagnosing the fault 
of the inverter. References [13-15] established the mixed logic dynamic model of motor and inverter, using the coordinate transformation to diagnose and locate IGBT fault.

Compared with the current-based inverter fault diagnosis methods, the inverter fault voltage-based methods have many advantages, such as simple use and low cost, and, in order to reduce the influence of unknown disturbance in motor driven system, extended state observer, which is based on the mixed logic dynamic model, is used to improve robustness of fault diagnostic method.

The remainder of this paper is organized as follows. The mixed logic model of the motor and inverter is introduced in Section 2. The schematic of inverter IGBT open-circuit fault diagnosis is analyzed in Section 3, as well as obtaining the output phase voltage of the circuit under normal working and fault conditions. The feasibility and effectiveness of the proposed method are verified by the simulation test in Section 4. Finally, a conclusion and some perspectives are proposed.

\section{The Mixed Logic Dynamic Model of the Inverter}

The topology diagram of the motor driving system is shown in Figure 1. The inverter circuit is composed of six IGBTs $T_{1} \sim T_{6}$ and six reverse diodes $D_{1} \sim D_{6}$. $\mathrm{U}_{\mathrm{dc}}$ is the voltage of the DC side, $\left(i_{a}, i_{b}, i_{c}\right)$ are the three-phase winding current. $\left(e_{a}\right.$, $\left.e_{b}, e_{c}\right)$ are opposite electromotive forces, $R$ is the stator winding resistor, and $L$ is the stator inductor. $F_{1} \sim F_{6}$ are fast fuses that convert short-circuit fault into open-circuit fault [2], so this paper only studies the diagnostic method of open-circuit fault.

In the motor drive system, the motor is equivalent to a circuit consisting of a series of resistor $R$, inductor $L$, and opposite electromotive forces $e$. The formula of the phase voltage of the motor is shown as

$$
\left\{\begin{array}{l}
u_{a n}=R i_{a}+L \frac{d i_{a}}{d t}+e_{a}, \\
u_{b n}=R i_{b}+L \frac{d i_{b}}{d t}+e_{b}, \\
u_{c n}=R i_{c}+L \frac{d i_{c}}{d t}+e_{c},
\end{array}\right.
$$

where $u_{a n}, u_{b n}$, and $u_{c n}$ are the voltage between the threephase winding of the motor and the hypothetical midpoint $n$ of the circuit.

According to the characteristics of the circuit, the formulas are shown as

$$
\begin{gathered}
\left\{\begin{array}{l}
u_{a n}=u_{a o}+u_{o n}, \\
u_{b n}=u_{b o}+u_{o n}, \\
u_{c n}=u_{c o}+u_{o n},
\end{array}\right. \\
i_{a}+i_{b}+i_{c}=0,
\end{gathered}
$$

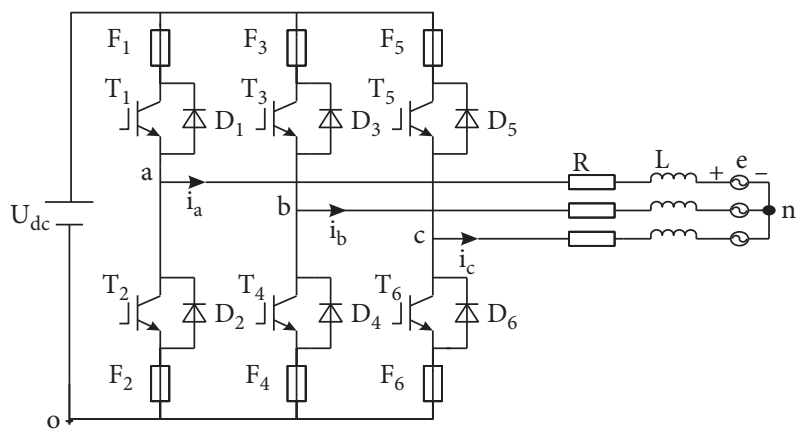

FIGURE 1: The topology diagram of the motor driving system.

$$
e_{a}+e_{b}+e_{c}=0
$$

Based on (1)-(4), the phase voltage is shown as

$$
\left[\begin{array}{l}
u_{a n} \\
u_{b n} \\
u_{c n}
\end{array}\right]=\frac{1}{3}\left[\begin{array}{ccc}
2 & -1 & -1 \\
-1 & 2 & -1 \\
-1 & -1 & 2
\end{array}\right]\left[\begin{array}{l}
u_{a o} \\
u_{b o} \\
u_{c o}
\end{array}\right]
$$

In practice, three or more IGBTs fault of the inverter will not occur at the same time. This paper discusses the diagnosis method of a single IGBT or two IGBTs open-circuit fault. Taking the $c$-phase as an example, the model of the inverter is established by the operating state of $u_{c o}$ when IGBTs work normally or abnormally.

Due to the change of current flow direction and the on/ off signals change of IGBTs in the motor driving system, the operating state of $u_{c o}$ can be affected. The normal current flow path of the $c$-phase IGBT is shown in Figure 2, where $s_{1} \sim s_{6}$ are the on/off signals of the 6 IGBTs of the inverter, $s_{i}(i=1,2, . ., 6)=1$ shows that IGBT is on, and $s_{i}(i=1,2, .$. , $6)=0$ shows that IGBT is off. $\left(\delta_{a}, \delta_{b}, \delta_{c}\right)$ are the current flow direction of the three-phase winding of the motor, $\delta_{i}(i=a, b$, $c)=1$ shows that current flows into the $i$-phase, and $\delta_{i}(i=a$, $b, c)=0$ shows that current does not flow into the $i$-phase.

Figure 2 shows the operation state of $u_{c o}$. The results are shown in Table 1 . The logical operation of the state of $u_{c o}$ is performed in Table 1 making $u_{\text {co }} \neq 0$ by logical OR operation.

So, the formula of $u_{c o}$ is shown as follows:

$$
u_{c o}=U_{d c}\left(s_{5} \bar{s}_{6} \delta_{c}+s_{5} \bar{s}_{6} \bar{\delta}_{c}+\bar{s}_{5} \bar{s}_{6} \bar{\delta}_{c}\right)=U_{d c} \bar{s}_{6}\left(s_{5}+\bar{s}_{5} \bar{\delta}_{c}\right) \text {. }
$$

When the inverter works normally, the formulas of $u_{a o}$, $u_{b o}$, and $u_{c o}$ are shown as

$$
\left\{\begin{array}{l}
u_{a o}=U_{d c} \overline{s_{2}}\left(s_{1}+\overline{s_{1}} \bar{\delta}_{a}\right), \\
u_{b o}=U_{d c} \overline{s_{4}}\left(s_{3}+\overline{s_{3}} \bar{\delta}_{b}\right), \\
u_{c o}=U_{d c} \overline{s_{6}}\left(s_{5}+\overline{s_{5}} \bar{\delta}_{c}\right) .
\end{array}\right.
$$

Based on (5) and (7), the three-phase voltage is shown as follows: 


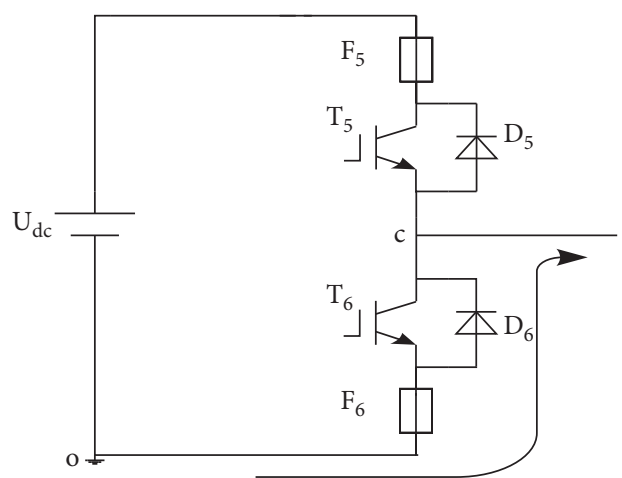

(a)

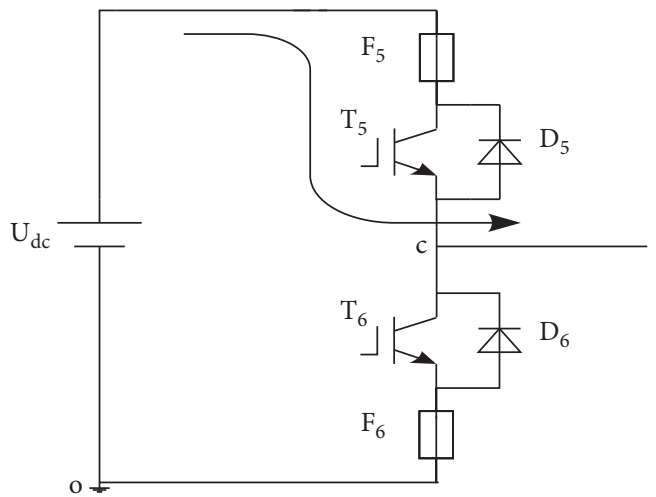

(c)

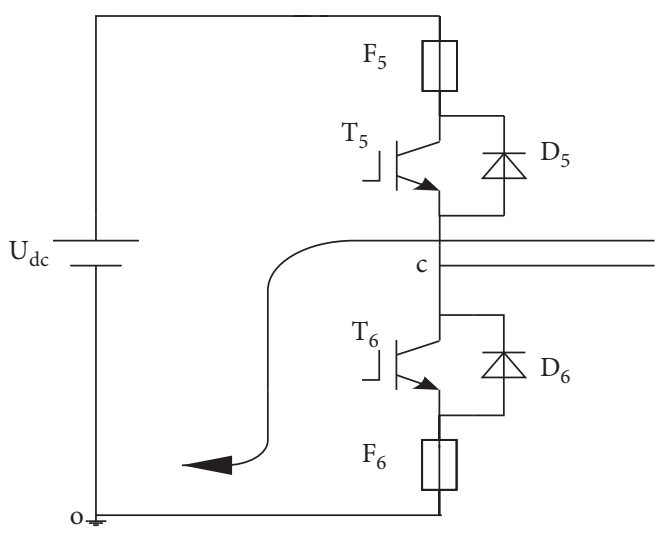

(e)

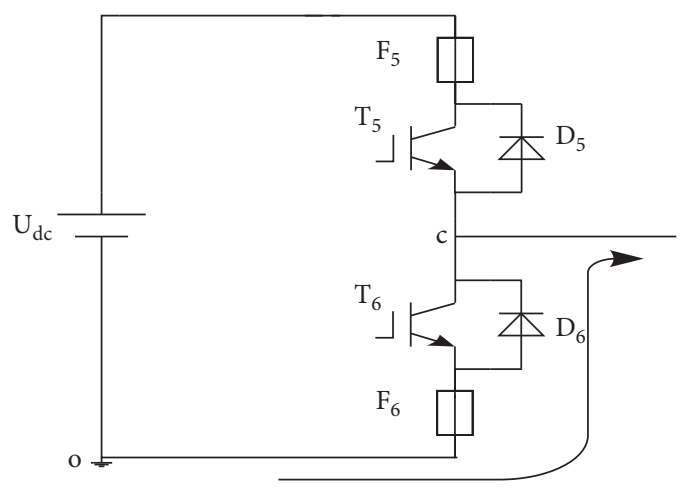

(b)

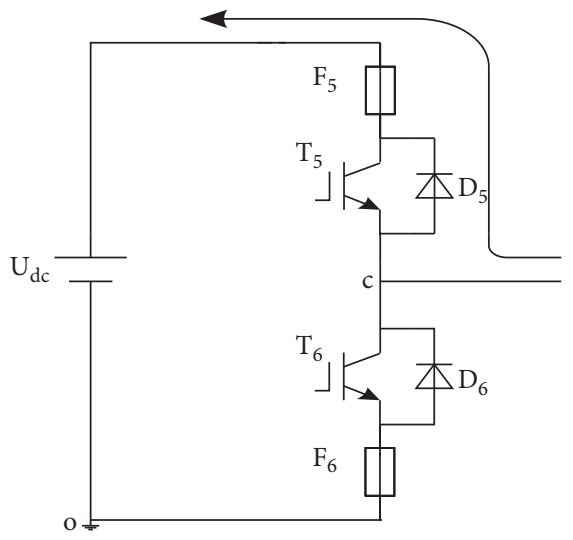

(d)

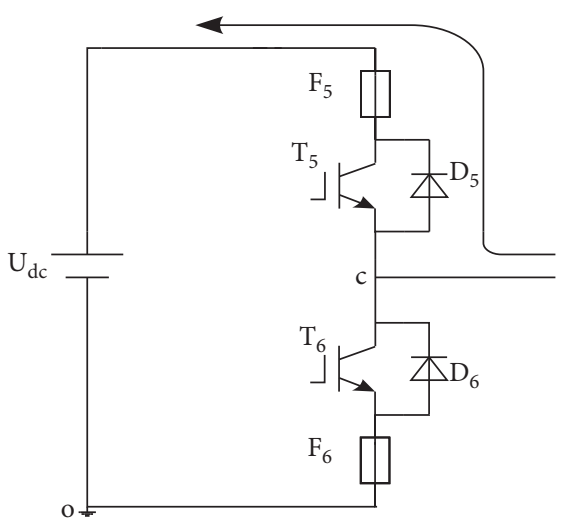

(f)

Figure 2: The current flow path of the $c$-phase IGBT when working normally.

TABLE 1: The truth table of $u_{\text {co }}$ when IGBTs are working normally.

\begin{tabular}{lccc}
\hline$s_{5}$ & $s_{6}$ & $\delta_{c}$ & \\
\hline 0 & 0 & 1 & $u_{c o}$ \\
0 & 1 & 1 & 0 \\
1 & 0 & 1 & 0 \\
0 & 0 & 0 & $U_{d c}$ \\
0 & 1 & 0 & $U_{d c}$ \\
1 & 0 & 0 & 0 \\
\hline
\end{tabular}




$$
\left[\begin{array}{l}
u_{a n} \\
u_{b n} \\
u_{c n}
\end{array}\right]=\frac{U_{d c}}{3}\left[\begin{array}{ccc}
2 & -1 & -1 \\
-1 & 2 & -1 \\
-1 & -1 & 2
\end{array}\right]\left[\begin{array}{l}
\bar{s}_{2}\left(s_{1}+\bar{s}_{1} \bar{\delta}_{a}\right) \\
\bar{s}_{4}\left(s_{3}+\bar{s}_{3} \bar{\delta}_{b}\right) \\
\bar{s}_{6}\left(s_{5}+\bar{s}_{5} \bar{\delta}_{c}\right)
\end{array}\right]
$$

Substituting (8) into (1), the hybrid logic dynamic model of the inverter is established as

$$
\left[\begin{array}{c}
\dot{i}_{a} \\
\dot{i}_{b} \\
\dot{i}_{c}
\end{array}\right]=\left[\begin{array}{ccc}
-\frac{R}{L} & 0 & 0 \\
0 & -\frac{R}{L} & 0 \\
0 & 0 & -\frac{R}{L}
\end{array}\right]+\frac{U_{d c}}{3 L}\left[\begin{array}{ccc}
2 & -1 & -1 \\
-1 & 2 & -1 \\
-1 & -1 & 2
\end{array}\right]\left[\begin{array}{l}
\bar{s}_{2}\left(s_{1}+\bar{s}_{1} \bar{\delta}_{a}\right) \\
\bar{s}_{4}\left(s_{3}+\bar{s}_{3} \bar{\delta}_{b}\right) \\
\bar{s}_{6}\left(s_{5}+\bar{s}_{5} \bar{\delta}_{c}\right)
\end{array}\right]+\left[\begin{array}{rrr}
-\frac{1}{L} & 0 & 0 \\
0 & -\frac{1}{L} & 0 \\
0 & 0 & -\frac{1}{L}
\end{array}\right]\left[\begin{array}{l}
e_{a} \\
e_{b} \\
e_{c}
\end{array}\right] .
$$

Equation (9) can be written in matrix form as

$$
i=A i+B_{1} \sigma+B_{2} e,
$$

where $\quad i=\left[\begin{array}{l}i_{a} \\ i_{b} \\ i_{c}\end{array}\right], \quad \delta=\left[\begin{array}{l}\bar{s}_{2}\left(s_{1}+\bar{s}_{1} \bar{\delta}_{a}\right) \\ \bar{s}_{4}\left(s_{3}+\bar{s}_{3} \bar{\delta}_{b}\right) \\ \bar{s}_{6}\left(s_{5}+\bar{s}_{5} \bar{\delta}_{c}\right)\end{array}\right], \quad e=\left[\begin{array}{c}e_{a} \\ e_{b} \\ e_{c}\end{array}\right]$, $A=\left[\begin{array}{ccc}-R / L & 0 & 0 \\ 0 & -R / L & 0 \\ 0 & 0 & -R / L\end{array}\right], B_{1}=U_{d c} / 3 L\left[\begin{array}{ccc}2 & -1 & -1 \\ -1 & 2 & -1 \\ -1 & -1 & 2\end{array}\right]$, and $B_{2}=\left[\begin{array}{ccc}-1 / L & 0 & 0 \\ 0 & -1 / L & 0 \\ 0 & 0 & -1 / L\end{array}\right]$.

\section{The Diagnosis Method of Open-Circuit Fault in the Inverter}

3.1. The Voltage Expansion Observer. In reality, there are a lot of disturbances in the inverter system, so the voltage extended observer is designed to estimate the output three-phase voltage in real time, thus eliminating the influence of some other unknown disturbances and uncertainties in the system and improving the accuracy of fault diagnosis.

$$
\left\{\begin{array}{l}
x^{(n)}(t)=f\left(x, x^{(1)}, \ldots, x^{(n-1)}, t\right)+\omega(t)+b u \\
y=x(t)
\end{array}\right.
$$

where $x(t)$ is the system state variable, $y(t)$ is the system output variable, $f\left(x, x^{(1)}, \ldots, x^{(n-1)}, t\right)$ is an unknown function, $\omega(t)$ is an unknown disturbance, and $b$ is the gain of the input variable $u$.

Treating $f\left(x, x^{(1)}, \ldots, x^{(n-1)}, t\right)$ and $\omega(t)$ as additional state variables, the expansion observer of the system can be shown as

$$
\left\{\begin{array}{l}
\dot{z_{1}}=z_{2}-g_{1}\left(z_{1}-x(t)\right), \\
\cdots \\
\dot{z_{n}}=z_{n+1}-g_{n}\left(z_{1}-x(t)\right)+b u \\
\dot{z_{n+1}}=-g_{n+1}\left(z_{1}-x(t)\right) .
\end{array}\right.
$$

In (12), the system uses $x(t)$ as input to track the expanded state variables $x(t), x^{(1)}(t), \ldots, x^{(n-1)}(t), x^{(n)}(t)$. So $z_{1} \longrightarrow x(t), \ldots, z_{n} \longrightarrow x^{(n-1)}(t), z_{n+1} \longrightarrow a(t), a(t)=f\left(x, x^{(1)}\right.$, $\left.x^{(\mathrm{n}-1)}, t\right)+\omega(t) \cdot g_{i}($.$) is a nonlinear function and it is gen-$ erally taken as

$$
\begin{aligned}
\operatorname{fal}(\varepsilon, \alpha, \delta) & = \begin{cases}|\mathcal{\varepsilon}|^{\alpha} \operatorname{sat}(\varepsilon), & |\varepsilon|>\delta, \\
\operatorname{sign}(\varepsilon), & |\varepsilon| \leq \delta,\end{cases} \\
\operatorname{sat}(\varepsilon) & = \begin{cases}\frac{\varepsilon}{\varsigma} & |\varepsilon| \leq \varsigma, \\
\operatorname{sign}(\varepsilon), & |\varepsilon|>\varsigma .\end{cases}
\end{aligned}
$$

Rewrite the hybrid logic dynamic model (10) of the inverter as

$$
\dot{i}=A i+B_{1} \sigma+B_{2} e=A i+B\left(B_{1} \sigma\right)+B_{2} e=A i+B u+B_{2} e,
$$

where $B=\operatorname{diag}(3)$ and $u=B_{1} \sigma=\left[u_{a n}, u_{b n}, u_{c n}\right]^{T}$.

When the extended observer is designed according to equation (3.8), its expression is

$$
\left\{\begin{array}{l}
\dot{z_{1}}=z_{2}-\beta_{1} \mathrm{fal}\left(\varepsilon, \alpha_{1}, \delta_{1}\right)+f_{0}\left(z_{1}\right)+b u_{1}, \\
\dot{z_{2}}=-\beta_{2} \operatorname{fal}\left(\varepsilon, \alpha_{2}, \delta_{2}\right)
\end{array}\right.
$$

where $z_{1}$ estimates the three-phase current $i, z_{2}$ estimates three-phase voltage disturbance of motor drive system, $\beta_{1}$ and $\beta_{2}$ are observer coefficients, $\alpha_{1}$ and $\alpha_{2}$ are nonlinear factors, $\delta_{1}$ and $\delta_{2}$ are filter factors, $\varepsilon=z 1-\mathrm{i}, \mathrm{f}_{0}(\mathrm{z} 1)=\mathrm{Az}_{1}$, $b=B_{2}$, and $u_{1}=e$.

\subsection{Diagram of Open-Circuit Fault Diagnosis}

3.2.1. The Open-Circuit Fault of Single IGBT. Take $c$-phase IGBT single-phase open-circuit fault as an example. When only open-circuit faults occur in $T_{5}$, the flow channel of the current is shown in Figure 3. The dotted line is the current direction under different switching modes. 


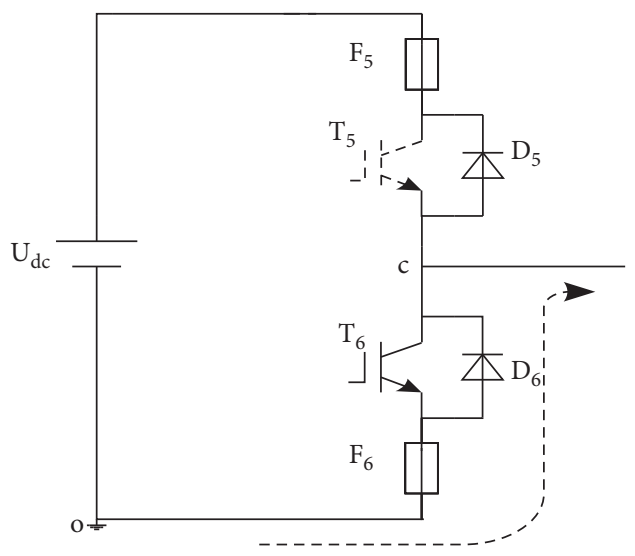

(a)

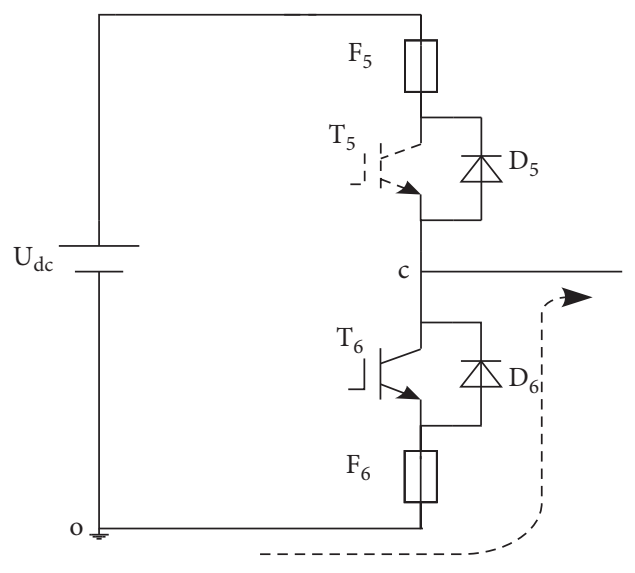

(c)

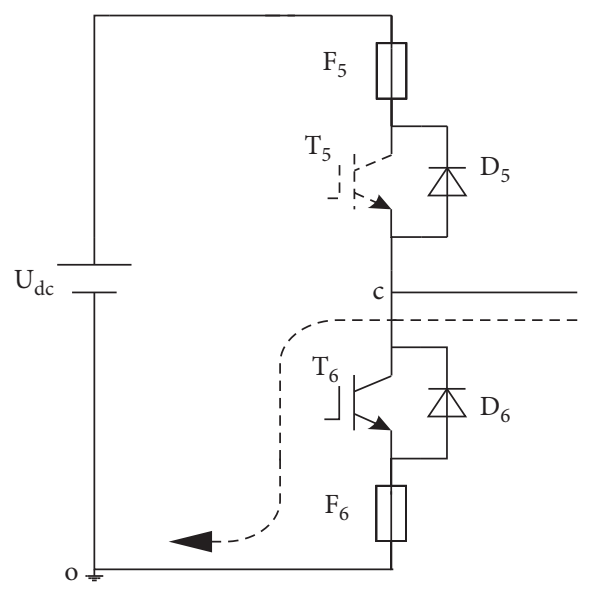

(e)

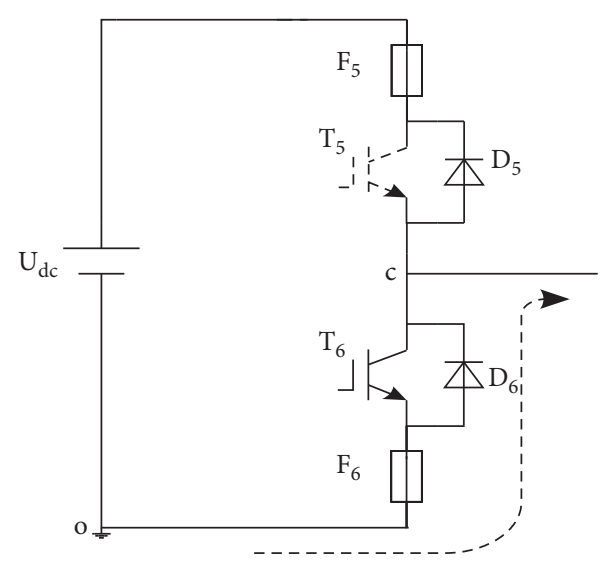

(b)

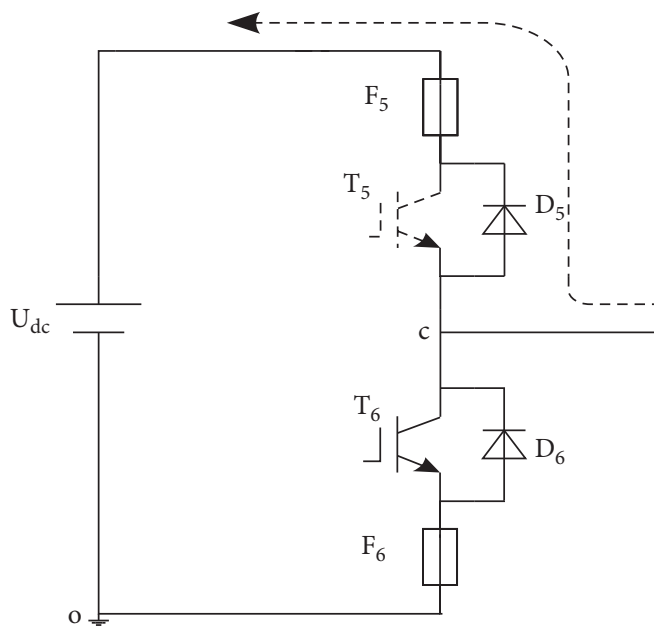

(d)

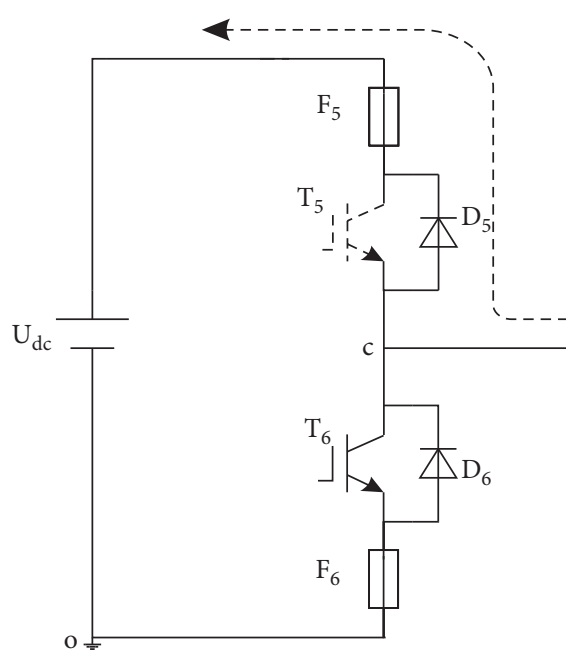

(f)

Figure 3: The flow path of the current when IGBT open-circuit fault only occurs in $T_{5}$.

If the open-circuit fault occurs in $T_{5}, u_{c o}$ is different from (7), $u_{c o}^{\prime}$ denotes the open-circuit fault voltage, and its truth table is shown in Table 2.

Based on Table 2, $u_{c o}^{\prime}$ can be calculated by logical operation as

$$
u_{c o}^{\prime}=U_{d c}\left(\bar{s}_{5} \bar{s}_{6} \bar{\delta}_{c}+s_{5} \bar{s}_{6} \bar{\delta}_{c}\right)=U_{d c} \bar{s}_{6} \bar{\delta}_{c} .
$$

When the open-circuit fault occurs in $T_{5}$, the formulas of $u_{a n}, u_{b n}$, and $u_{c n}^{\prime}$ are shown as 
TABLe 2: The truth table of $u_{c o}^{\prime}$ when the open-circuit fault only occurs in $T_{5}$.

\begin{tabular}{cccc}
\hline$s_{5}$ & $s_{6}$ & $\delta_{c}$ & $u_{c o}^{\prime}$ \\
\hline 0 & 0 & 1 & 0 \\
0 & 1 & 1 & 0 \\
1 & 0 & 1 & 0 \\
0 & 0 & 0 & $U_{d c}$ \\
0 & 1 & 0 & 0 \\
1 & 0 & 0 & $U_{d c}$ \\
\hline
\end{tabular}

$$
\left[\begin{array}{c}
u_{a n} \\
u_{b n} \\
u_{c n}^{\prime}
\end{array}\right]=\frac{U_{d c}}{3}\left[\begin{array}{ccc}
2 & -1 & -1 \\
-1 & 2 & -1 \\
-1 & -1 & 2
\end{array}\right]\left[\begin{array}{c}
\bar{s}_{2}\left(s_{1}+\bar{s}_{1} \bar{\delta}_{a}\right) \\
\bar{s}_{4}\left(s_{3}+\bar{s}_{3} \bar{\delta}_{b}\right) \\
\bar{s}_{6} \bar{\delta}_{c}
\end{array}\right] .
$$

Based on (10) and (18), when the open-circuit fault occurs in $T_{5}$, the formulas of voltage residual are shown as

$$
\left\{\begin{array}{l}
\Delta u_{a n}=-\frac{U_{d c}}{3} s_{5} \overline{s_{6}} \delta_{c} \\
\Delta u_{b n}=-\frac{U_{d c}}{3} s_{5} \overline{s_{6}} \delta_{c} \\
\Delta u_{c n}=\frac{2 U_{d c}}{3} s_{5} \overline{s_{6}} \delta_{c}
\end{array}\right.
$$

Equation (19) shows that when the open-circuit fault occurs in $T_{5}$, we can obtain $\triangle u_{a n}=\Delta u_{b n} \leq 0, \triangle u_{c n} \geq 0$, and $\Delta u_{c n}=2 \Delta u_{a n}=2 \Delta u_{b n}$.

\subsubsection{Two IGBTs Occur in an Open-Circuit Fault. Two} IGBTs in a phase

When the open-circuit fault occurs in $T_{5}$ and $T_{6}$ at the same time, the current flow path is as shown in Figure 4. The results of $u_{c o}^{\prime}$ are shown in Table 3 .

The logical operation of $u_{c o}^{\prime \prime}$ is shown in Table 3 , making $u_{c o}^{\prime \prime} \neq 0$ by logical OR operation. So $u_{c o}^{\prime \prime}$ is shown as

$$
u_{c o}^{\prime \prime}=U_{d c}\left(\bar{s}_{5} \bar{s}_{6} \bar{\delta}_{c}+\bar{s}_{5} s_{6} \bar{\delta}_{c}+s_{5} \bar{s}_{6} \bar{\delta}_{c}\right)=U_{d c} \bar{\delta}_{c} .
$$

When the open-circuit fault occurs in $T_{5}$ and $T_{6}$ at the same time, $u_{a \eta}, u_{b n}$, and $u_{c n}^{\prime}$ are shown as

$$
\left[\begin{array}{c}
u_{a n} \\
u_{b n} \\
u_{c n}^{\prime \prime \prime}
\end{array}\right]=\frac{U_{d c}}{3}\left[\begin{array}{ccc}
2 & -1 & -1 \\
-1 & 2 & -1 \\
-1 & -1 & 2
\end{array}\right]\left[\begin{array}{c}
\bar{s}_{2}\left(s_{1}+\bar{s}_{1} \bar{\delta}_{a}\right) \\
\bar{s}_{4}\left(s_{3}+\bar{s}_{3} \bar{\delta}_{b}\right) \\
\bar{\delta}_{c}
\end{array}\right] .
$$

Based on (10) and (21), when the open-circuit fault occurs in $T_{5}$ and $T_{6}$ at the same time, the voltage residual is shown as

$$
\left\{\begin{array}{l}
\Delta u_{a n}=-\frac{U_{d c}}{3}\left[s_{5} \overline{s_{6}}+\overline{\delta_{c}}\left(\overline{s_{5} s_{6}}-1\right)\right] \\
\Delta u_{b n}=-\frac{U_{d c}}{3}\left[s_{5} \overline{s_{6}}+\overline{\delta_{c}}\left(\overline{s_{5} s_{6}}-1\right)\right] \\
\Delta u_{c n}=\frac{2 U_{d c}}{3}\left[s_{5} \overline{s_{6}}+\overline{\delta_{c}}\left(\overline{s_{5} s_{6}}-1\right)\right]
\end{array}\right.
$$

From (22), the relationship of $\Delta u_{c n}, \Delta u_{a n}$, and $\Delta u_{b n}$ satisfied $\Delta u_{c n}=-2 \Delta u_{a n}=-2 \Delta u_{b n}$.

Two IGBTs in two phases

(1) Two IGBTs at the same position in two phases

Take the open-circuit fault of $T_{3}$ and $T_{5}$ as an example, and use the calculation method of the opencircuit fault of two switches in the same phase; the specific expressions of $u_{b o}^{\prime \prime \prime}$ and $u_{c o}^{\prime \prime \prime}$ can be obtained as

$$
\left\{\begin{array}{l}
u_{b o}^{\prime \prime \prime}=U_{d c} \overline{s_{4}} \overline{\delta_{b}}, \\
u_{c o}^{\prime \prime \prime}=U_{d c} \overline{s_{6}} \overline{\delta_{c}} .
\end{array}\right.
$$

When the open-circuit fault occurs in $T_{3}$ and $T_{5}$, the phase voltage of each phase is

$\left[\begin{array}{c}u_{a n} \\ u_{b n}^{\prime \prime \prime} \\ u_{c n}^{\prime \prime \prime}\end{array}\right]=\frac{U_{d c}}{3}\left[\begin{array}{ccc}2 & -1 & -1 \\ -1 & 2 & -1 \\ -1 & -1 & 2\end{array}\right]\left[\begin{array}{c}\bar{s}_{2}\left(s_{1}+\bar{s}_{1} \bar{\delta}_{a}\right) \\ \bar{s}_{4} \bar{\delta}_{b} \\ \bar{s}_{6} \bar{\delta}_{c}\end{array}\right]$.

According to (10) and (24), the residual voltage of $T_{3}$ and $T_{5}$ is

$$
\left\{\begin{array}{l}
\Delta u_{a n}=\frac{U_{d c}}{3}\left(-s_{3} \bar{s}_{4} \delta_{b}-s_{5} \bar{s}_{6} \delta_{c}\right), \\
\Delta u_{b n}=\frac{U_{d c}}{3}\left(2 s_{3} \bar{s}_{4} \delta_{b}-s_{5} \bar{s}_{6} \delta_{c}\right), \\
\Delta u_{c n}=\frac{U_{d c}}{3}\left(-s_{3} \bar{s}_{4} \delta_{b}+2 s_{5} \bar{s}_{6} \delta_{c}\right) .
\end{array}\right.
$$

According to (25), there is not a certain numerical relationship between the voltage residuals of the fault phases and those of the normal phase in a cycle, so it is impossible to locate the fault phases and the fault IGBTs by using the numerical relationship of voltage residuals.

However, the voltage residual value of the fault phase is still twice as the normal two-phase voltage in a certain cycle. For example, when the IGBTs $T_{3}$ and $T_{6}$ are normally on, the expression of the three-phase voltage residual is 


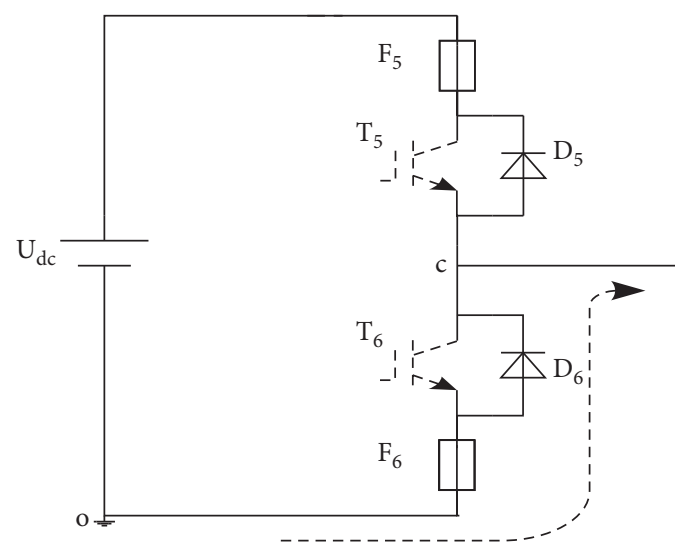

(a)

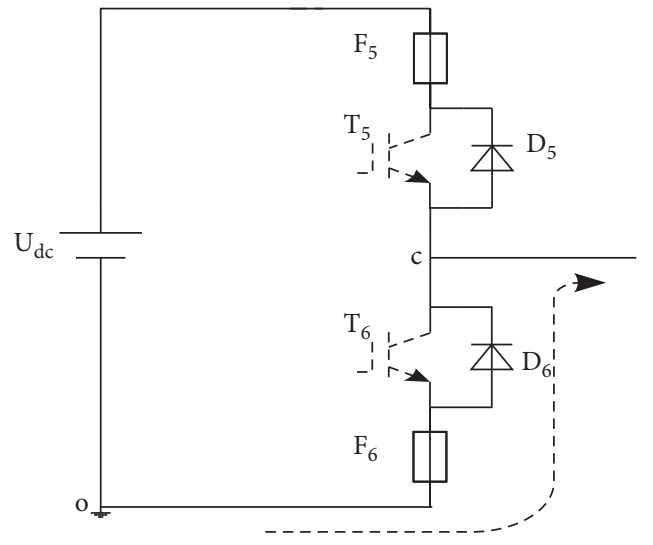

(c)

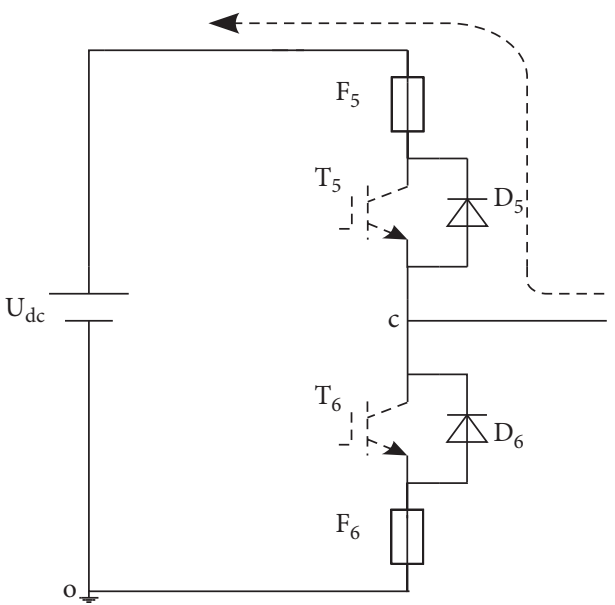

(e)

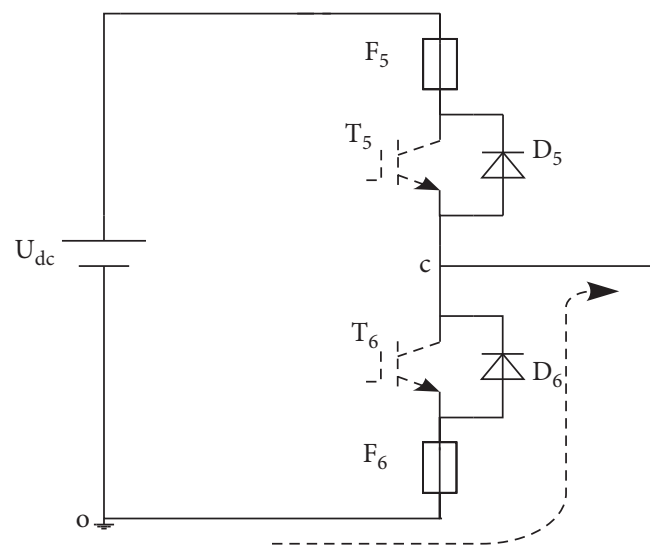

(b)

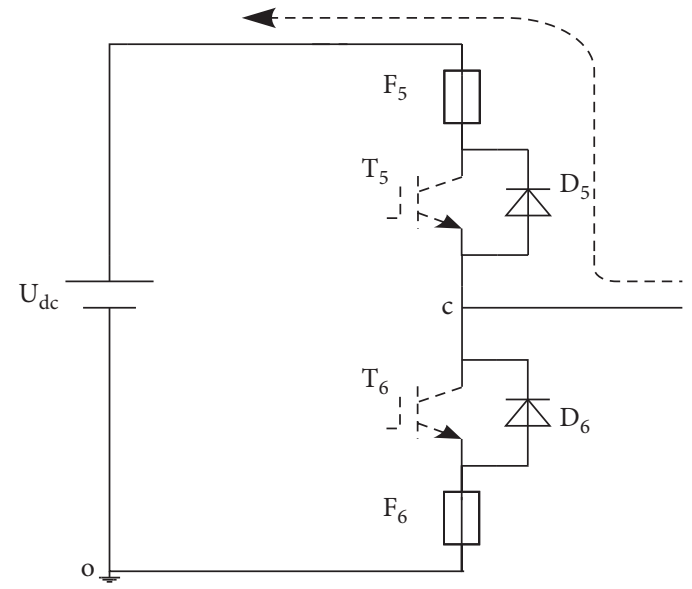

(d)

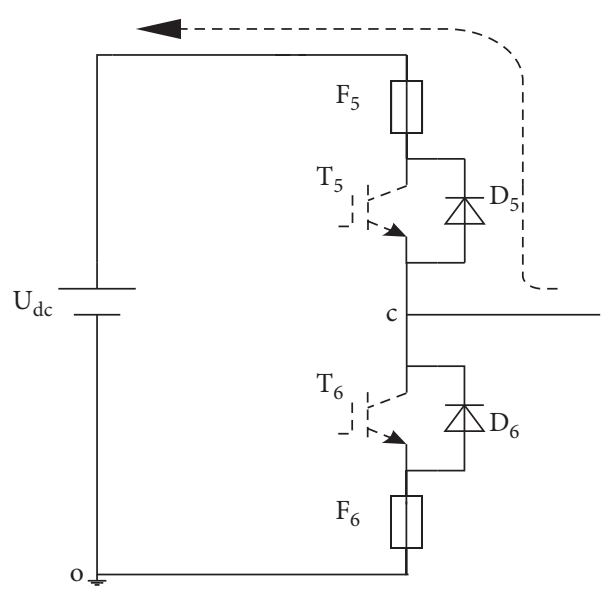

(f)

FIgURE 4: The current flow path when the open-circuit fault occurs in $T_{5}$ and $T_{6}$ at the same time.

TABLE 3: The truth table of $u_{c o}^{\prime \prime}$ when the open-circuit fault occurs in $T_{5}$ and $T_{6}$ at the same time.

\begin{tabular}{lccc}
\hline$s_{5}$ & $s_{6}$ & $\delta_{c}$ & $u_{c o}^{\prime \prime}$ \\
\hline 0 & 0 & 1 & 0 \\
0 & 1 & 1 & 0 \\
1 & 0 & 1 & 0 \\
0 & 0 & 0 & $U_{d c}$ \\
0 & 1 & 0 & $U_{d c}$ \\
1 & 0 & 0 & $U_{d c}$ \\
\hline
\end{tabular}




$$
\left\{\begin{array}{l}
\Delta u_{a n}=-\frac{U_{d c}}{3} s_{3} \bar{s}_{4} \delta_{b} \\
\Delta u_{b n}=\frac{2 U_{d c}}{3} s_{3} \bar{s}_{4} \delta_{b} \\
\Delta u_{c n}=-\frac{U_{d c}}{3} s_{3} \bar{s}_{4} \delta_{b}
\end{array}\right.
$$

When the IGBTs $T_{4}$ and $T_{5}$ are normally on, the three-phase voltage residual is

$$
\left\{\begin{array}{l}
\Delta u_{a n}=-\frac{U d c}{3} s_{5} \bar{s}_{6} \delta_{c} \\
\Delta u_{b n}=-\frac{U d c}{3} s_{5} \bar{s}_{6} \delta_{c} \\
\Delta u_{c n}=2 \frac{U_{d c}}{3} s_{5} \bar{s}_{6} \delta_{c}
\end{array}\right.
$$

When T4 and T5 are normal, the relationship between the IGBTs $T_{3}$ and $T_{5}$ is the same as that in the case of the open-circuit fault of the IGBT $T_{5}$.

(2) Two IGBTs at different position in two phases

Take the open-circuit fault of $T_{4}$ and $T_{5}$ of phase $b$ and $c$ as an example, and use the calculation method of the open-circuit fault of two switches in the same phase; $u_{c o}^{\prime \prime \prime \prime}$ and $u_{c o}^{\prime \prime \prime \prime}$ can be obtained as follows:

$$
\left\{\begin{array}{c}
u_{b o}^{\prime \prime \prime \prime}=U_{d c}\left(s_{3}+\bar{s}_{3} \bar{\delta}_{b}\right), \\
u_{c o}^{\prime \prime \prime \prime}=U_{d c} \bar{s}_{6} \bar{\delta}_{c},
\end{array}\right.
$$

and when the open-circuit fault occurs in $T_{4}$ and $T_{5}$, the phase voltage of each phase is

$$
\left[\begin{array}{c}
u_{a n} \\
u_{b n}^{\prime \prime \prime \prime} \\
u_{b n}^{\prime \prime \prime \prime}
\end{array}\right]=\frac{U_{d c}}{3}\left[\begin{array}{ccc}
2 & -1 & -1 \\
-1 & 2 & -1 \\
-1 & 2 & -1
\end{array}\right]\left[\begin{array}{c}
\bar{s}_{2}\left(s_{1}+\bar{s}_{1} \bar{\delta}_{a}\right) \\
s_{3}+\bar{s}_{3} \bar{\delta}_{b} \\
\bar{s}_{6} \bar{\delta}_{c}
\end{array}\right] .
$$

According to (10) and (29), the residual voltage of $T_{4}$ and $T_{5}$ is

$$
\left\{\begin{array}{l}
\Delta u_{a n}=\frac{U_{d c}}{3}\left[s_{4}\left(s_{3}+\bar{s}_{3} \bar{\delta}_{b}\right)-s_{5} \bar{s}_{6} \delta_{c}\right] \\
\Delta u_{b n}=\frac{U_{d c}}{3}\left[-2 s_{4}\left(s_{3}+\bar{s}_{3} \bar{\delta}_{b}\right)-s_{5} \bar{s}_{6} \delta_{c}\right] \\
\Delta u_{c n}=\frac{U_{d c}}{3}\left[s_{4}\left(s_{3}+\bar{s}_{3} \bar{\delta}_{b}\right)+2 s_{5} \bar{s}_{6} \delta_{c}\right] .
\end{array}\right.
$$

From (30), when the IGBTs $T_{4}$ and $T_{6}$ are normally on, the three-phase voltage residual is as follows:

$$
\left\{\begin{array}{l}
\Delta u_{a n}=\frac{U_{d c}}{3} s_{4}\left(s_{3}+\bar{s}_{3} \bar{\delta}_{b}\right) \\
\Delta u_{b n}=-\frac{2 U d c}{3} s_{4}\left(s_{3}+\bar{s}_{3} \bar{\delta}_{b}\right) \\
\Delta u_{c n}=\frac{U_{d c}}{3} s_{4}\left(s_{3}+\bar{s}_{3} \bar{\delta}_{b}\right)
\end{array}\right.
$$

In conclusion, the fault diagnosis method of single switch open-circuit fault and two switches in the same phase are the foundation of the fault diagnosis in the different phase, and the fault location of two switches in the different phase is a special case of single switch open-circuit fault. Therefore, this paper only simulates the open-circuit fault of a single switch and the open-circuit fault of two switches in the same phase.

3.3. Establishment of Voltage Residual Information Table. The above methods are used to deal with the open-circuit fault of phase $a$ and phase $b$, respectively. The residual voltage of phase $a$ and phase $b$ under different fault conditions can be obtained through calculation, and the results are shown in Table 4; and the open-circuit fault of a single switch and two switches in the same phase can be accurately located by looking up Table 4 .

3.4. The Diagnosis Strategy of Open-Circuit Fault in the Inverter. The flowchart of the inverter open-circuit fault diagnosis is shown in Figure 5. In this paper, it is judged whether the open-circuit fault occurs by comparing the voltage residual with the threshold. In an ideal case, the voltage residual of each phase of the inverter tends to zero. When the open-circuit fault of inverter occurs, the maximum absolute values of the voltage residuals of each phase are assumed to be $U_{T_{1}}, U_{T_{2}}, U_{T_{3}}, U_{T_{4}}, U_{T_{5}}$, and $U_{T_{6}}$. So, the threshold range is

$$
U_{\text {th }}=\frac{1}{2} \max \left\{U_{T_{1}}, U_{T_{2}}, U_{T_{3}}, U_{T_{4}}, U_{T_{5}}, U_{T_{6}}\right\}+\Delta,
$$

where $U_{t h}$ is the threshold and $\Delta$ is the safety margin, which is used to reduce the influence of measurement error, noise, and disturbance in the actual system.

\section{Simulation}

The simulation parameters of motor are given in Table 5 . When the inverter works normally, it suddenly changes the load value at $t=0.37 \mathrm{~s}$, the three-phase voltage output by the actual system, and the voltage extension observer, and the residuals between the actual voltage and the observed voltage are shown in Figures 6 and 7 .

From Figure 7, the voltage expansion observer is consistent with the actual system, and it is shown that that the observer has a good tracking effect.

During the simulation, the driving signal of $T_{5}$ is removed at $t=0.3 \mathrm{~s}$, and $t=0.37 \mathrm{~s}$ suddenly changes the load 
TABLE 4: The voltage residual information table.

\begin{tabular}{|c|c|c|}
\hline Bridge arm & $\begin{array}{l}\text { Fault switch } \\
\text { Normally }\end{array}$ & $\begin{array}{l}\text { Relationship between residuals } \\
\qquad \Delta u_{a n}=\Delta u_{b n}=\Delta u_{c n}\end{array}$ \\
\hline $\mathrm{a}$ & $\begin{array}{c}T_{1} \\
T_{2} \\
T_{1} \text { and } T_{2} \\
\end{array}$ & $\begin{array}{c}\Delta u_{a n}=-2 \Delta u_{b n}=-2 \Delta u_{c n} \geq 0 \\
\Delta u_{a n}=-2 \Delta u_{b n}=-2 \Delta u_{c n} \leq 0 \\
\Delta u_{a n}=-2 \Delta u_{b n}=-2 \Delta u_{c n}\end{array}$ \\
\hline b & $\begin{array}{c}T_{3} \\
T_{4} \\
T_{3} \text { and } T_{4} \\
\end{array}$ & $\begin{array}{c}\Delta u_{b n}=-2 \Delta u_{a n}=-2 \Delta u_{c n} \geq 0 \\
\Delta u_{b n}=-2 \Delta u_{a n}=-2 \Delta u_{c n} \leq 0 \\
\Delta u_{b n}=-2 \Delta u_{a n}=-2 \Delta u_{c n}\end{array}$ \\
\hline c & $\begin{array}{c}T_{5} \\
T_{6} \\
T_{5} \text { and } T_{6} \\
\end{array}$ & $\begin{array}{c}\Delta u_{c n}=-2 \Delta u_{a n}=-2 \Delta u_{b n} \geq 0 \\
\Delta u_{c n}=-2 \Delta u_{a n}=-2 \Delta u_{b n} \leq 0 \\
\Delta u_{c n}=-2 \Delta u_{a n}=-2 \Delta u_{b n}\end{array}$ \\
\hline
\end{tabular}

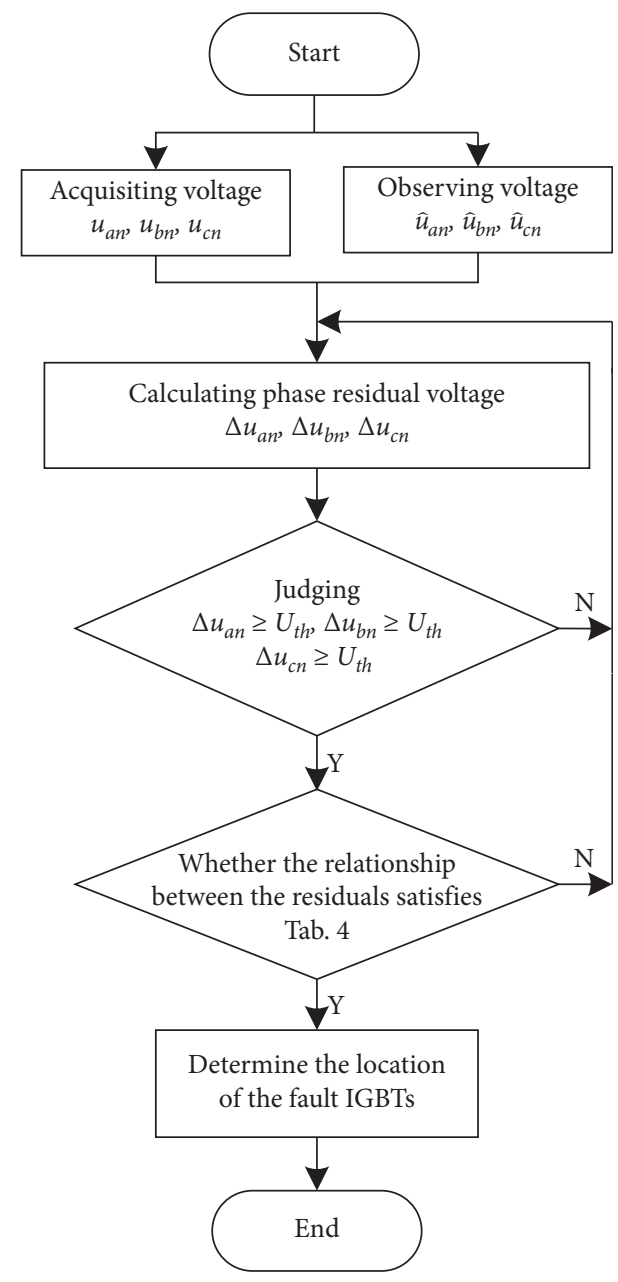

Figure 5: The flowchart of inverter open-circuit fault diagnosis.

TABLE 5: The inverter system parameters.

\begin{tabular}{lc}
\hline Description & Value \\
\hline Frequency $f$ & $50 \mathrm{~Hz}$ \\
DC side voltage $U_{d c}$ & $360 \mathrm{~V}$ \\
Stator resistance $R_{s}$ & $0.435 \Omega$ \\
Rotor resistance $R_{r}$ & $0.816 \Omega$ \\
Stator leakage inductance $L_{l s}$ & $4 \mathrm{mH}$ \\
Rotor leakage inductance $L_{r s}$ & $2 \mathrm{mH}$ \\
Mutual inductance $L_{m}$ & $69.31 \mathrm{mH}$ \\
Pole pair $p$ & 2 \\
Moment of inertia $J$ & $0.089 \mathrm{~kg} \cdot \mathrm{m}^{2}$ \\
\hline
\end{tabular}

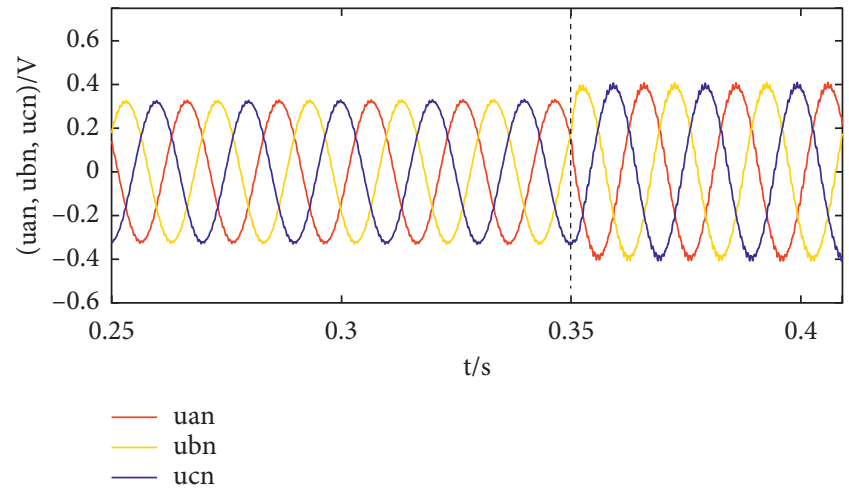

Figure 6: The normal three-phase voltage of the actual system and the voltage extension observer.

value. The three-phase voltage and voltage residual are shown in Figure 8 when the open-circuit fault occurs in $T_{5}$.

From Figure 8, the $c$-phase voltage is distorted, and the voltage residual rapidly increases in the positive direction after $0.3 \mathrm{~s}$. The voltage residuals in phase $a$ and phase $b$ negatively increase and the increasing trends of the two are approximately the same. The numerical relationship satisfies

$$
\Delta u_{c n}=-2 \Delta u_{b n}=-2 \Delta u_{a n} \geq 0 .
$$

By looking up the voltage residual information table in Table 4, the voltage residual exhibits the characteristics of the open-circuit fault in $T_{5}$. If $\Delta u_{c n}>U_{t h}$ is satisfied, the opencircuit fault occurs in $T_{5}$. After $0.37 \mathrm{~s}$, the system suddenly changes the load, and each phase amplitude of the voltage and voltage residual increase, but it can also detect that $T_{5}$ has an open-circuit fault. It shows that the method proposed in this paper has certain antinoise interference.

If the driving signals of $T_{5}$ and $T_{6}$ are removed at $t=0.3 \mathrm{~s}$ and $t=0.37 \mathrm{~s}$, the load suddenly changes. The three-phase voltage and voltage residual in which the open-circuit fault occurs in $T_{5}$ and $T_{6}$ are shown in Figure 9.

The proposed fault diagnosis method is compared with other fault diagnosis methods; and the objector (for openloop system, closed-loop system, or both), diagnosis time, robustness, cost, and fault type are the comparison index, and they are shown in Table 6. From Table 6, the proposed fault diagnosis method has good performance compared with other fault methods. 


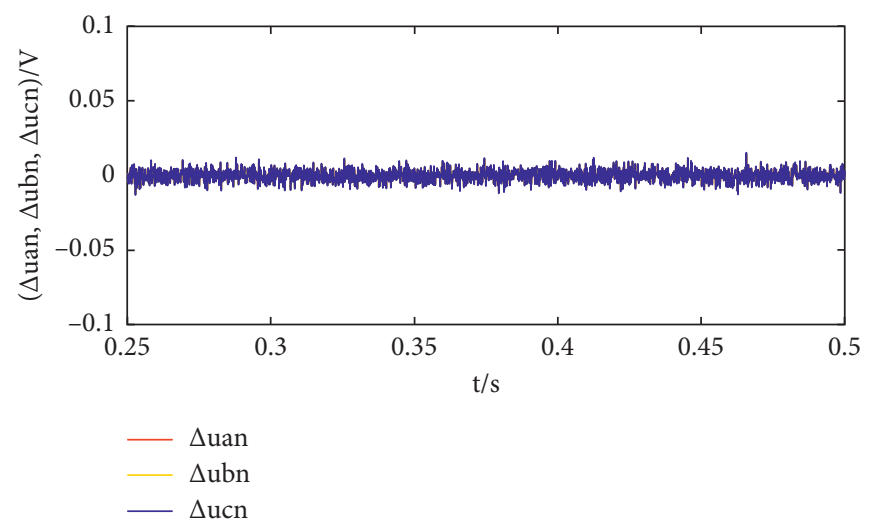

FIGURE 7: The residuals between the actual voltage and the observed voltage.

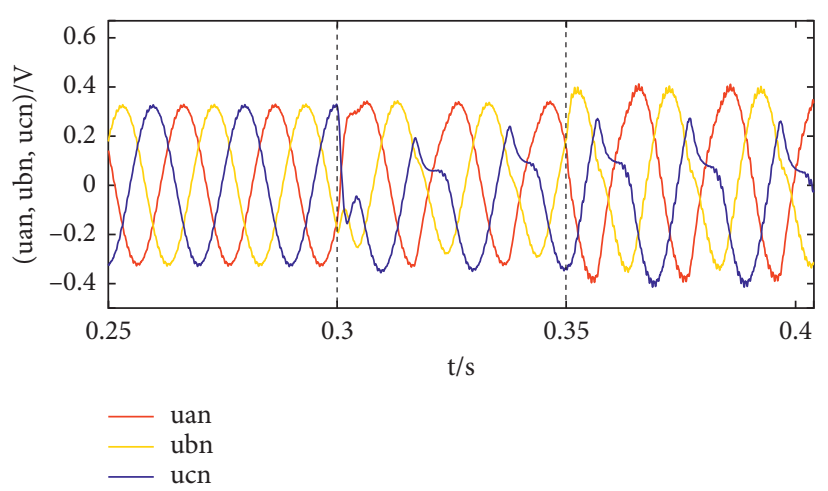

(a)

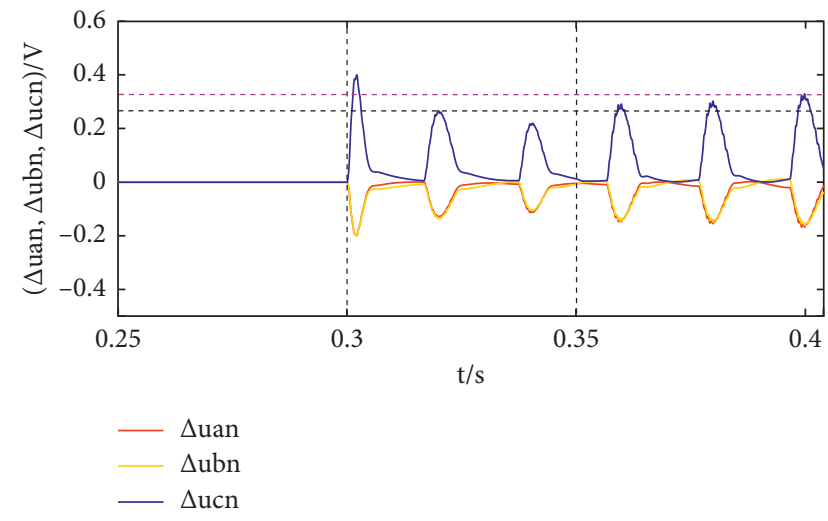

(b)

FIgURE 8: The three-phase voltage and voltage residual when the open-circuit fault occurs in $T_{5}$. (a) The three-phase voltage when the opencircuit fault occurs in $T_{5}$. (b) The voltage residual when the open-circuit fault occurs in $T_{5}$.

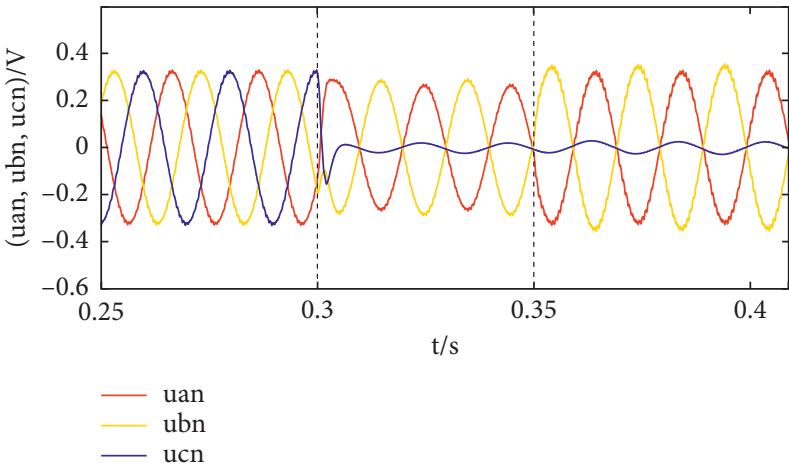

(a)

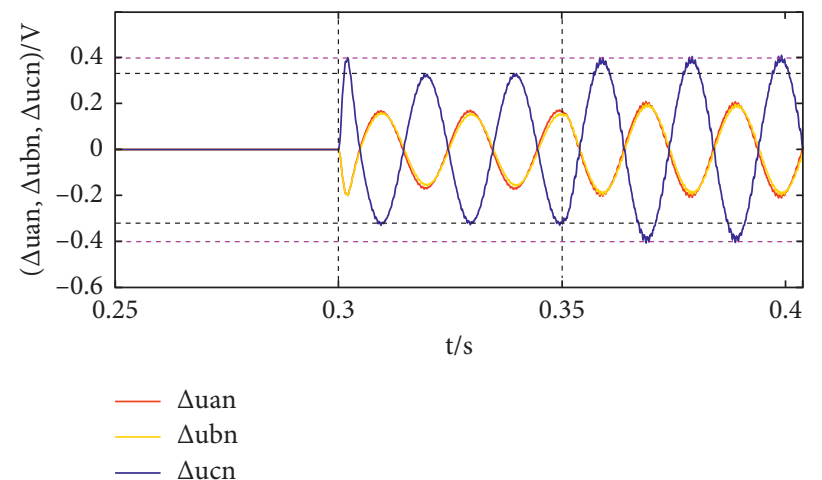

(b)

FIgURE 9: The three-phase voltage and voltage residual when the open-circuit fault occurs in $T_{5}$ and $T_{6}$ at the same time. (a) The three-phase voltage when the open-circuit fault occurs in $T_{5}$ and $T_{6}$ at the same time. (b) The voltage residual when the open-circuit fault occurs in $T_{5}$ and $T_{6}$ at the same time.

TABLE 6: Comparison of the proposed fault diagnosis method and other fault methods.

\begin{tabular}{|c|c|c|c|c|c|}
\hline Fault diagnosis method & Objector & Diagnosis time & Robustness & Cost & Fault type \\
\hline The neural network method & Both & More than one cycle & Low & Low & Single \\
\hline The fuzzy logic method & Both & More than one cycle & Medium & High & Multiple \\
\hline Park's vector approach & Open-loop & More than one cycle & Low & Low & Single \\
\hline Spectrum analysis method & Both & More than one cycle & Medium & High & Single \\
\hline The proposed method & Both & About $1 / 3$ cycle & High & Low & Multiple \\
\hline
\end{tabular}




\section{Conclusion}

In this paper, the mixed logic dynamic model of the inverter is established by the flow path of current when the inverter works normally and abnormally; and considering the dead time of the switching signal in the modeling process avoids the misdiagnosis of the fault. At the same time, a voltage expansion observer is designed to avoid the problem of increasing the fault rate by introducing other hardware circuits. The simulation results show that the method can be accurately located and has a short diagnosis time. In the future, it is possible to study the diagnostic method of the inverter of two IGBTs in different phases, where the opencircuit fault occurs at the same time, and add a certain faulttolerant strategy to ensure that the inverter can work normally.

\section{Data Availability}

The inverter system parameters data used to support the findings of this study are included within the article.

\section{Conflicts of Interest}

The authors declare that there are no conflicts of interest regarding the submission of this manuscript, and the manuscript is approved by all the authors for publication.

\section{Acknowledgments}

This study was supported by the National Natural Science Foundation of China (nos. 61573282 and 61833013), the Key Research and Development Program (Key Projects) of Shaanxi Province (no. 2019KWZ-10), and the Key Research and Development Program (General Projects) of Shaanxi Province (no. 2020GY-194).

\section{References}

[1] J. Lamb and B. Mirafzal, "Open-circuit IGBT fault detection and location isolation for cascaded multilevel converters," IEEE Transactions on Industrial Electronics, vol. 64, no. 6, pp. 4846-4856, 2017.

[2] S. Ravyts, G. V. D. Broeck, L. Hallemans, M. D. Vecchia, and J. Driesen, "Fuse-based short-circuit protection of converter controlled low-voltage DC grids," IEEE Transactions on Power Electronics, vol. 35, no. 11, pp. 11694-11706, 2020.

[3] L. Wen, X. Li, L. Gao et al., "A new convolutional neural network-based data-driven fault diagnosis method," IEEE Transactions on Industrial Electronics, vol. 65, no. 7, pp. 5990-5998, 2017.

[4] K.-H. Chao, L.-Y. Chang, and F.-Q. Xu, "Smart fault-tolerant control system based on chaos theory and extension theory for locating faults in a three-level T-type inverter," Applied Sciences, vol. 9, no. 15, p. 3071, 2019.

[5] L. Xu, M. Cao, B. Song, J. Zhang, Y. Liu, and F. E. Alsaadi, "Open-circuit fault diagnosis of power rectifier using sparse autoencoder based deep neural network," Neurocomputing, vol. 311, pp. 1-10, 2018.

[6] H. Yan, Y. Xu, F. Cai et al., "PWM-VSI fault diagnosis for a PMSM drive based on the fuzzy logic approach," IEEE
Transactions on Power Electronics, vol. 34, no. 1, pp. 759-768, 2018.

[7] R. Muzzammel, Restricted Boltzmann Machines Based Fault Estimation in Multi Terminal HVDC Transmission system, in Proceedings of the International Conference on Intelligent Technologies and Applications, pp. 772-790, Bahawalpur, Pakistan, November 2019.

[8] I. Bandyopadhyay, P. Purkait, and C. Koley, "Performance of a classifier based on time-domain features for incipient fault detection in inverter drives," IEEE Transactions on Industrial Informatics, vol. 15, no. 1, pp. 3-14, 2018.

[9] F. Husari and J. Seshadrinath, "Inter-turn fault diagnosis of induction motor fed by PCC-VSI using Park vector approach," in Proceedings of the IEEE international conference on power electronics, drives and energy systems (PEDES), pp. 1-6, IEEE, Jaipur, Rajasthan, December 2020.

[10] J. A. P. Sánchez, D. U. Campos-Delgado, D. R. Espinoza-Trejo et al., "Fault diagnosis in grid-connected PV NPC inverters by a model-based and data processing combined approach," IET Power Electronics, vol. 12, no. 12, pp. 3254-3264, 2019.

[11] J. H. Song and K. H. Kim, "Fast detection of open-switch fault using moving filter for a PWM VSI-fed PMSM drive system," International Journal of Applied Engineering Research, vol. 12, pp. 932-939, 2017.

[12] X. Ge, J. Pu, B. Gou et al., "An open-circuit fault diagnosis approach for single-phase three-level neutral-point-clamped converters," IEEE Transactions on Power Electronics, vol. 33, no. 3, pp. 2559-2570, 2017.

[13] M. Skowron, M. Wolkiewicz, T. Orlowska-Kowalska, and C. Kowalski, "Application of self-organizing neural networks to electrical fault classification in induction motors," Applied Sciences, vol. 9, no. 4, p. 616, 2019.

[14] J. Lu, H. Li, Y. Liu, and F. Li, "Survey on semi-tensor product method with its applications in logical networks and other finite-valued systems," IET Control Theory \& Applications, vol. 11, no. 13, pp. 2040-2047, 2017.

[15] Y. Zhai, N. Zhong, and Z. Zhang, "A fault detection and identification method based on mixed logic dynamic model for three-phase inverter using single current sensor," in Proceedings of the CAA symposium on fault detection, supervision and safety for technical processes (SAFEPROCESS), pp. 362-367, IEEE, Xiamen, China, July 2019. 